3-1-2019

\title{
General Error-based Active Disturbance Rejection Control for Swift Industrial Implementations
}

\author{
R. Madonski \\ Southeast University, Nanjing
}

S. Shao

Cleveland State University, s.shao@csuohio.edu

H. Zhang

Cleveland State University

Z. Gao

Cleveland State University, Z.GAO@csuohio.edu

J. Yang

Southeast University, Nanjing

See next page for additional authors

Follow this and additional works at: https://engagedscholarship.csuohio.edu/scimath_facpub

Part of the Mathematics Commons

How does access to this work benefit you? Let us know!

\section{Repository Citation}

Madonski, R.; Shao, S.; Zhang, H.; Gao, Z.; Yang, J.; and Li, S., "General Error-based Active Disturbance Rejection Control for Swift Industrial Implementations" (2019). Mathematics Faculty Publications. 332.

https://engagedscholarship.csuohio.edu/scimath_facpub/332

This Article is brought to you for free and open access by the Mathematics and Statistics Department at EngagedScholarship@CSU. It has been accepted for inclusion in Mathematics Faculty Publications by an authorized administrator of EngagedScholarship@CSU. For more information, please contact library.es@csuohio.edu. 


\section{Authors}

R. Madonski, S. Shao, H. Zhang, Z. Gao, J. Yang, and S. Li 


\title{
General error-based active disturbance rejection control for swift industrial implementations
}

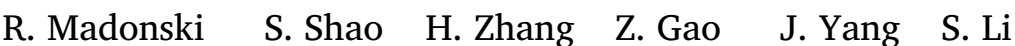

\begin{abstract}
A B S T R A C T
In this article, a typical 2DOF active disturbance rejection control (ADRC) design is restructured into a 1DOF form, thus making it compatible with standard industrial control function blocks and enhancing its market competitiveness. This methodology integrates the previously separated components, such as the profile generator, state observer, feedback controller, feedforward terms, and disturbance rejection, into one unified structure. In doing so, certain ADRC components can be made simpler (or even obsolete) without sacrificing the nominal control performance, which further simplifies the control synthesis and tuning. A generalized version of the errordriven design is adopted and rigorously proved here using the singular perturbation theory. The experimental verification of the utilized approach is carried out using a disturbed DC-DC buck converter.
\end{abstract}

\section{Introduction}

For active disturbance rejection control (ADRC Gao, 2006; Han, 2009), which is emerging as a viable general solution for industrial control, a natural question arises about optimization of its practical implementation. With a track record of successful deployments, including power (Lotfi, Zomorodi, \& Landers, 2016; Wu, Sun, \& Lee, 2017), motion (Chu, Sun, Wu, \& Sepehri, 2018; Cortes-Romero, JimenezTriana, Coral-Enriquez, \& Sira-Ramirez, 2017; Michalek \& Kielczewski, 2017), and process control (Madonski, Nowicki, \& Herman, 2014; Zheng, Chen, \& Gao, 2009), the aspiration now is to make ADRC schemes even simpler in terms of design and tuning, thus making them more competitive in the industrial control market. By comparison, most of the industrial control algorithms, whether already incorporated in programmable logic controllers (PLCs) or available as standalone solutions, are implemented as single blocks in one degree-of-freedom (1DOF) error-driven forms. Such design, most likely resulting from the omnipresent use of PID, favors practitioners, since its compact design eases implementation and commission as well as simplifies training of the control personnel.

On the other hand, in the majority of existing ADRC-based techniques, a 2DOF design is commonly adopted, in which the outputbased inner-loop extracts the lumped information about the uncertain/unmodeled parts of the dynamics (denoted as total disturbance) by means of an extended state observer (ESO) and on-line cancels their effect on the controlled signal, while the outer-loop governs the remaining simplified system. Although such approach is often desired in control research, as it allows to design, tune, and analyze controller and observer parts separately, it may not be appealing for control practitioners, as it requires implementation and commission of several, interconnected structures. There have been studies focused on seamless integration of already existing 1DOF controllers (mostly PID-type) with the ADRC methodology (e.g. Herbst, 2016; Madonski, Gao, \& Lakomy, 2015; Madonski, Nowicki, \& Herman, 2016; Nowak et al., 2018; Xue, Madonski, Lakomy, Gao, \& Huang, 2017). It was shown that such transition is possible and beneficial, but may be relatively troublesome for first-time users, as they may not be familiar with the disturbance rejection-based control design.

Such dissonance in design methodologies preemptively creates a barrier for ADRC, which slows down its industrial expansion. In order to eliminate it, a methodology of constructing a 1DOF ADRC solution can be used. Typical elements seen in ADRC schemes (like profile generator, state observer, feedforward and feedback controller) can be encapsulated into a single block, representing solely the relation between the tracking error and the control signal. It is made possible by expressing the control objective, not in the typical output-based form, but in the error-based form, a procedure that has been independently developed in Michalek (2016) and Zhang (2017). The introduced error-driven form not only gives the ability to restructure the typical ADRC into commonly seen industrial forms, but also enables it to be implemented in specialized embedded settings, in which only specific 1DOF forms of governors can be utilized - for example see Fig. 1. 


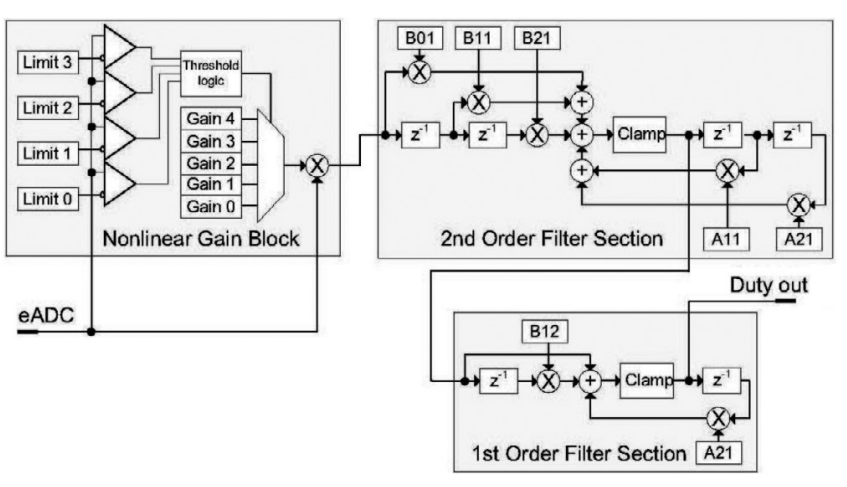

Fig. 1. Diagram of a digital compensator in PWM control scheme in TI-UCD9224 chip, which only accepts controllers implemented as three-pole three-zero transfer functions (Using the UCD9224EVM-464 - user's guide, 2017).

The overall idea is to make the implementation of ADRC resembling that of currently used industrial solutions (like PID), hence making it easier to deploy in real applications or to swiftly replace the existing controller. Desirably, the goal is to make the single-block ADRC design a "plug\&play" industrial solution, thus allowing its wider utilization in practice. In this work, a linearized and parametrized version of ADRC, as seen in Gao (2003), is considered as a nominal case of an output-based ADRC, from which the error-based version will be derived. Its choice is justified by practical convenience, but supported with numerous works, in which this simplified design was successful in governing even complex, nonlinear systems (Godbole, Kolhe, \& Talole, 2013; Guerra, Arteaga-Perez, Gutierrez-Giles, \& Maya-Ortiz, 2016; Madonski, Kordasz, \& Sauer, 2014; Ramirez-Neria, Sira-Ramirez, Garrido-Moctezuma, \& Luviano-Juarez, 2014; Wang, Li, Wang, \& Li, 2017).

An additional practical advantage of expressing the ADRC in errorbased form is that in cases of trajectory tracking with unavailable reference time-derivatives (through measurement or a'priori knowledge), these unknown signals can be treated as part of the total disturbance and their effects can be estimated by the ESO (Michalek, 2016; Zhang, 2017). Such approach makes tools like differentiators obsolete, thus greatly simplifying the control design procedure, especially for highorder systems. It seems that such methodology corresponds to the very spirit of the ADRC paradigm, which makes uncertainty consideration a priority (Gao, 2014). This particular feature of ADRC has been previously used (e.g. Dai, Yang, Wang, \& Li, 2017; Xue \& Huang, 2015, 2018), here however, its comprehensive analysis is given. This, together with conducted theoretical, numerical, and experimental validation, is considered to be the main contribution of this work.

The rest of the article is organized as follows. First, some illustrative examples of the error-based ADRC scheme are shown in Section 2. A numerical comparison is also performed here in order to highlight the properties of both the typical output-based and the considered errorbased ADRC designs. Next section presents the generalized methodology for developing the 1DOF compact ADRC design (with its rigorous theoretical analysis in the Appendix). In Section 4, hardware verification of the proposed compact scheme is carried out for a challenging control scenario based on reference voltage trajectory tracking in a buck power converter. Section 5 concludes the work.

\section{Error-based ADRC design: specific case}

The idea of 1DOF ADRC in error-based form is shown first through a case study using a generic second order $(n=2)$ plant model, which can represent a wide range of physical systems with (practically) acceptable accuracy (Fliess \& Join, 2013). The control objective is to make the system output $y(t) \in \mathbb{R}$ track a reference trajectory $r(t) \in \mathbb{R}$ by manipulating the input signal $u(t) \in \mathbb{R}$. The goal is to globally minimize the trajectory tracking error $(e(t) \triangleq r(t)-y(t))$ in the presence of unknown system dynamics and unpredictable external disturbance. Both system input and output signals are available for controller synthesis. Additionally, $r(t)$ satisfies following assumptions:

A1. it is bounded and only known at current time instant $t$,

A2. its consecutive reference time-derivatives $(\dot{r}, \ddot{r}, \ddot{r})$ exist for all $t \geq 0$ and are bounded,

A3. it is not known in advance, nor its consecutive reference timederivatives. ${ }^{1}$

\subsection{Illustrative example}

A generic control-affine second order system model is given as:

$\Psi^{(2)}: \quad \ddot{y}=\bar{f}+b_{0} u$,

where $b_{0} \neq 0$ is the system uncertain input gain and $\bar{f} \in \mathbb{R}$ represents a lumped combination of unknown dynamic terms, resulting from both internal and external disturbances. Simple modification of (1) allows to express the same system as:

$\Psi^{(2)}: \quad \ddot{y}=\bar{f}+b_{0} u+\hat{b}_{0} u-\hat{b}_{0} u,=f+\hat{b}_{0} u$,

where $\hat{b}_{0} \neq 0$ is a rough approximation of $b_{0}$ and $f=\bar{f}+\left(b_{0}-\hat{b}_{0}\right) u$ now also includes the uncertainty related to the input gain, hence it will be referred to as the system total disturbance (Gao, 2014).

Instead of typical output-based approach of choosing state variables as $\boldsymbol{x}=\left[\begin{array}{lll}x_{1} & x_{2} & x_{3}\end{array}\right]=\left[\begin{array}{lll}y & \dot{y} & f\end{array}\right]$, seen in typical ADRC designs for second order plant models, here the state variables are chosen by analyzing the tracking error definition:

$$
\begin{aligned}
& \triangleq \triangleq r-y=x_{1}, \\
& \dot{e} \triangleq \dot{r}-\dot{y}=\dot{x}_{1}=x_{2}, \\
\Sigma^{(2)}: & \ddot{e} \triangleq \ddot{r}-\ddot{y}=\ddot{x}_{1}=\dot{x}_{2} \stackrel{(2)}{=} \underbrace{\ddot{r}-f}_{f^{*}}-\hat{b}_{0} u=x_{3}-\hat{b}_{0} u .
\end{aligned}
$$

As seen above, the goal is to choose a set of phase state variables that would allow to incorporate the otherwise unavailable term $\ddot{\mathrm{r}}$ as part of the extended state variable, hence treat it as part of the total disturbance ( $x_{3}=f^{*} \in \mathbb{R}$ ), to be estimated and compensated in the disturbance rejection loop (designed later).

Remark 1. By reformulating the control task in error-based form, the objective now is to stabilize the new output signal (e) at zero with the use of the same control input $(u)$, despite the influence of matched total disturbance $\left(f^{*}\right)$.

The system (1) can thus be written in alternative coordinates $\boldsymbol{x}=$ $\left[\begin{array}{lll}x_{1} & x_{2} & x_{3}\end{array}\right]=\left[\begin{array}{lll}e & \dot{e} & f^{*}\end{array}\right]$ in virtually extended form as:

$\Sigma^{(3)}:\left\{\begin{array}{l}\dot{x}_{1}=x_{2}, \\ \dot{x}_{2}=x_{3}-\hat{b}_{0} u, \\ \dot{x}_{3}=\dot{f}^{*},\end{array}\right.$

where $\dot{f}^{*}=\dddot{r}-\dot{f}$. It is assumed here, and throughout this work, that $f^{*}$ is time-differentiable and $\dot{f}^{*}$ is bounded. ${ }^{2}$ For the above system expressed in error-based form, a corresponding $(n+m)$ th order ESO, for the considered plant model order $n=2$ and assumed polynomial-type disturbance model order $m=1$, can be proposed as:

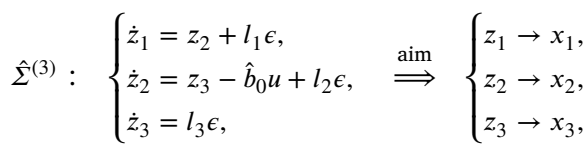

1 This emulates a practical scenario in which the reference trajectory results from a current action of an outer control system (e.g. cascade control) or from a current interaction with a different system (e.g. multi-subsystems plant).

2 The validity of this assumption in both theoretical and practical considerations has been studied extensively, for example, in Shao and Gao (2017) and Xue and Huang $(2015,2018)$. 
where $z=\left[z_{1} z_{2} z_{3}\right]=\left[\hat{x}_{1} \hat{x}_{2} \hat{x}_{3}\right]$ is the vector of state variables estimates, and $\epsilon \triangleq e-z_{1} \equiv e-\hat{e}$ is the observer estimation error. The main purpose of this observer is to timely and precisely reconstruct the information about the acting total disturbance $\left(z_{3} \rightarrow f^{*}\right)$, which is essential for its cancellations. Proper selection of gains $\left\{l_{1}, l_{2}, l_{3}\right\}>0$ using the notion of observer bandwidth $\left(\omega_{o}>0\right)$ will be discussed later.

The application of control action in form of: $u \triangleq\left(u_{0}+f^{*}\right) / \hat{b}_{0}$ to the system model (3), would reduce its dynamics to an idealized integral chain $\ddot{e}=-u_{0}$. In practice however, one would have to be satisfied with either:

$u=\frac{u_{0}+\hat{f}^{*}}{\hat{b}_{0}}=\frac{k_{0} z_{1}+k_{1} z_{2}+z_{3}}{\hat{b}_{0}} \quad$ or $\quad u=\frac{u_{0}+\hat{f}^{*}}{\hat{b}_{0}}=\frac{k_{0} x_{1}+k_{1} x_{2}+z_{3}}{\hat{b}_{0}}$,

which choice by the control designer depends on the noisiness of the tracking error $(e)$ and/or its derivative $(\dot{e})$. Proper selection of gains $\left\{k_{0}, k_{1}\right\}>0$ using the notion of controller bandwidth $\left(\omega_{c}>0\right)$ will be discussed later.

Remark 2. The above control actions can be seen as special cases of conventional output-based ADRC with additional feed-forward, but with the unknown terms estimated here on-line by the observer.

Combining left-hand side proposition from (6) with (5) gives:

$\dot{z}_{1}=z_{2}+l_{1}\left(e-z_{1}\right)=-l_{1} z_{1}+z_{2}+l_{1} e$,

$\dot{z}_{2}=z_{3}-\left(k_{0} z_{1}+k_{1} z_{2}+z_{3}\right)+l_{2}\left(e-z_{1}\right)=\left(-k_{0}-l_{2}\right) z_{1}-k_{1} z_{2}+l_{2} e$,

$\dot{z}_{3}=l_{3}\left(e-z_{1}\right)=-l_{3} z_{1}+l_{3} e$,

which in state-space can be expressed as:

$\dot{z}=\boldsymbol{A} \boldsymbol{z}+\boldsymbol{l} e$, where: $\boldsymbol{z}=\left[\begin{array}{l}z_{1} \\ z_{2} \\ z_{3}\end{array}\right], \boldsymbol{A}=\left[\begin{array}{ccc}-l_{1} & 1 & 0 \\ -l_{2}-k_{0} & -k_{1} & 0 \\ -l_{3} & 0 & 0\end{array}\right], \boldsymbol{l}=\left[\begin{array}{l}l_{1} \\ l_{2} \\ l_{3}\end{array}\right]$.

The transfer functions $T_{1}(s)$ (from $E(s)$ to $Z_{1}(s)$ ), $T_{2}(s)$ (from $E(s)$ to $Z_{2}(s)$ ), and $T_{3}(s)$ (from $E(s)$ to $Z_{3}(s)$ ) can be calculated as:

$\boldsymbol{T}=\boldsymbol{C}(s \boldsymbol{I}-\boldsymbol{A})^{-1} \boldsymbol{l}$, where: $\boldsymbol{T}=\left[\begin{array}{c}T_{1}(s) \\ T_{2}(s) \\ T_{3}(s)\end{array}\right]=\left[\begin{array}{c}\frac{Z_{1}(s)}{E(s)} \\ \frac{Z_{2}(s)}{E(s)} \\ \frac{Z_{3}(s)}{E(s)}\end{array}\right], \boldsymbol{C}=\left[\begin{array}{ccc}1 & 0 & 0 \\ 0 & 1 & 0 \\ 0 & 0 & 1\end{array}\right]$,

which allows to write the entire ADRC structure for second order system (1) in a single, error-driven, compact transfer function:

$$
\begin{aligned}
G_{\mathrm{ADRC}}(s)=\frac{U(s)}{E(s)} & =\frac{k_{0} T_{1}(s)+k_{1} T_{2}(s)+T_{3}(s)}{\hat{b}_{0}} \\
& =\frac{\left(k_{0} l_{1}+k_{1} l_{2}+l_{3}\right) s^{2}+\left(k_{0} l_{2}+k_{1} l_{3}\right) s+k_{0} l_{3}}{\hat{b}_{0}\left[s^{2}+\left(l_{1}+k_{1}\right) s+l_{1} k_{1}+l_{2}+k_{0}\right] s} .
\end{aligned}
$$

\subsection{Illustrative example (special case)}

From analyzing the example in Section 2.1, it can be notices that in the considered compact version of ADRC, the desired error dynamics for the closed-loop system can be prescribed in the ESO already during its design process, thus eliminating a need for constructing a dedicated outer-loop feedback controller. ${ }^{3}$

In order to do that, the exemplary second order system model in error-based form (3) can be artificially supplemented with term $\eta=$ $k_{0} e+k_{1} \dot{e}$, which gives:

$$
\ddot{e}=\ddot{r}-f-\hat{b}_{0} u+(\eta-\eta)
$$

3 Such methodology corresponds to the proposition seen in Michalek (2016). It has been advocated there, however, that since both reference $(r)$ and output (y) are often available in practice at current time, it is thus reasonable to keep proportional action $u_{0}=k_{0} e=k_{0}(r-y)$ in order to robustify the control design in the presence of estimation errors, inevitable in real applications.
Table 1

Comparison between the conventional 2DOF output- and the considered 1DOF error-based ADRC for the considered exemplary second order system.

\begin{tabular}{lll}
\hline Criteria & output-based ADRC & error-based ADRC \\
\hline Objective interpretation & traj. follow. $(y \rightarrow r)$ & zero stab. $(|e| \rightarrow 0)$ \\
Control form & $u\left(e, z_{2}, z_{3}, \dot{r}, \dot{r}\right)$ & $u\left(e, z_{3}\right)$ \\
System/Observer form & $x=\left[y \dot{y} f^{*}\right], \dot{z}(z, u, y)$ & $\boldsymbol{x}=\left[e \dot{e} f^{*}\right], \dot{z}(z, u, e)$ \\
Total disturbance form & $f^{*}(y, \dot{y}, u)$ & $f^{*}(y, \dot{y}, \dot{r}, \dot{r}, u)$ \\
Extra assumptions & $\dot{r}, \dot{r}$ available & - \\
Tuning parameters & $\omega_{c}, \omega_{o}, \hat{b}_{0}$ & $\omega_{c}, \omega_{o}, \hat{b}_{0}$ \\
Assumed models & $n=2, m=1$ & $n=2, m=1$ \\
\hline
\end{tabular}

$\bar{\Sigma}^{(2)}: \quad k_{0} e+k_{1} \dot{e}+\ddot{e}=\underbrace{\ddot{r}-f+k_{0} e+k_{1} \dot{e}}_{f^{*}}-\hat{b}_{0} u$.

The above system can be written with same set of coordinates $x=$ $\left[\begin{array}{lll}x_{1} & x_{2} & x_{3}\end{array}\right]=\left[\begin{array}{lll}e & \dot{e} & f^{*}\end{array}\right]$ as (cf.(4)):

$\bar{\Sigma}^{(3)}:\left\{\begin{array}{l}\dot{x}_{1}=x_{2}, \\ \dot{x}_{2}=x_{3}-k_{0} x_{1}-k_{1} x_{2}-\hat{b}_{0} u, \\ \dot{x}_{3}=\dot{f}^{*}\end{array}\right.$

for which the linear ESO takes the form (cf.(5)):

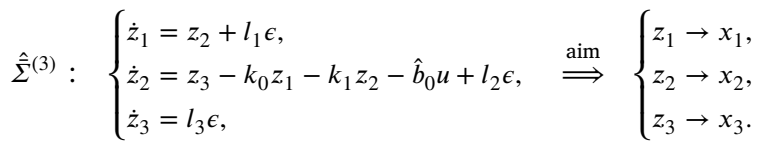

For the modified system model (12), the control signal can be proposed simply as disturbance rejection action $u=z_{3} / \hat{b}_{0}$, but when applied to (12) gives the same desired closed-loop dynamics $\ddot{e}+k_{1} \dot{e}+k_{0} e=0$, as seen in the previous example. It means that this case is (theoretically) analogous to the one seen in Section 2.1, and the transition to the compact ADRC would result in the same transfer function $G_{\mathrm{ADRC}}(s)$ seen in (10), but with different practical ramifications.

\subsection{Comparison analysis}

It is important now to show qualitatively and quantitatively the differences between the typical output-based ADRC and the considered error-based compact version. Table 1 compares two approaches using several qualitative criteria. From the table, one can notice the disparity in assumptions about the availability of certain signals for control synthesis. The quantitative comparison, on the other hand, is performed here in both time and frequency domains in Matlab/Simulink using a second order system $G(s)=b /\left(s^{2}+a_{1} s+a_{2}\right)$, with $a_{1}=8, a_{2}=15$, and $b=1$.

Time-domain analysis. Three disturbance rejection control schemes are quantitatively compared in time-domain, namely output-based ADRC, output-based ADRC with extra feed-forward (FF) term, ${ }^{4}$ and the compact error-based ADRC. The derivation of the latter is done as shown in Section 2.1. Tuning parameters are set the same for all three cases: $\hat{b}_{0}=1, \omega_{c}=40$ (controller bandwidth), and $\omega_{o}=4 \omega_{c}$ (observer bandwidth). The ability to recover performance is tested by applying an input-additive step disturbance at $t=0.6 \mathrm{~s}$. The results of simulations are collectively presented in Fig. 2. From the figures, one can notice that the error-based ADRC has similar performance in terms of error minimization to the output-based ADRC with FF (which coincides with Remark 2). It was expected that the output-based ADRC without feedforward would have the worst performance out of the tested cases, since it is not supplemented with any additional information that could potentially unburden the ESO. Interestingly enough, all three cases have similar performance in case of disturbance rejection.

\footnotetext{
4 Since it is assumed (for practical reasons) that the time derivatives of the reference signal $(\dot{r}, \ddot{r})$ are not available for controller synthesis, the needed terms are computed on-line with two tracking differentiators (TD) (Han, 2009), based on the known reference signal $(r)$.
} 

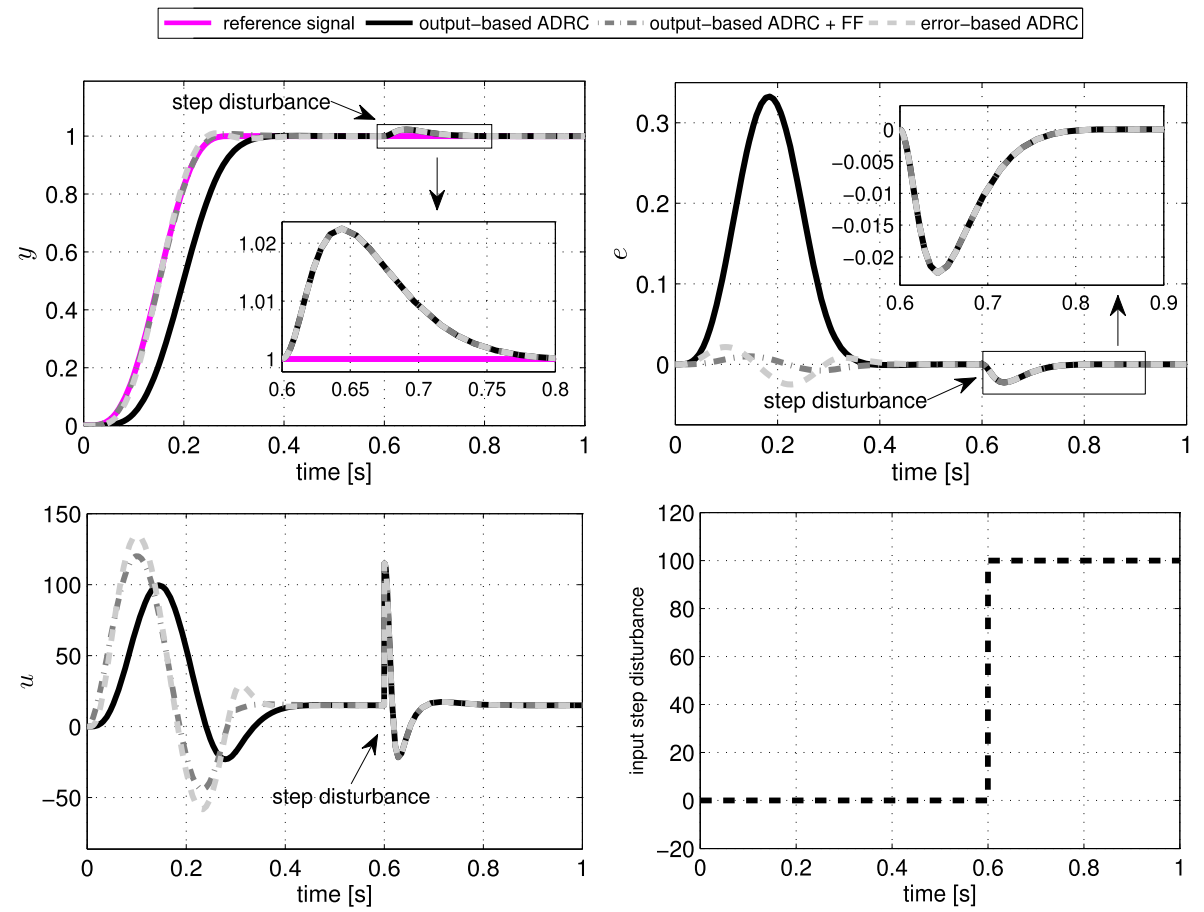

Fig. 2. Time-domain quantitative comparison of different ADRC designs.
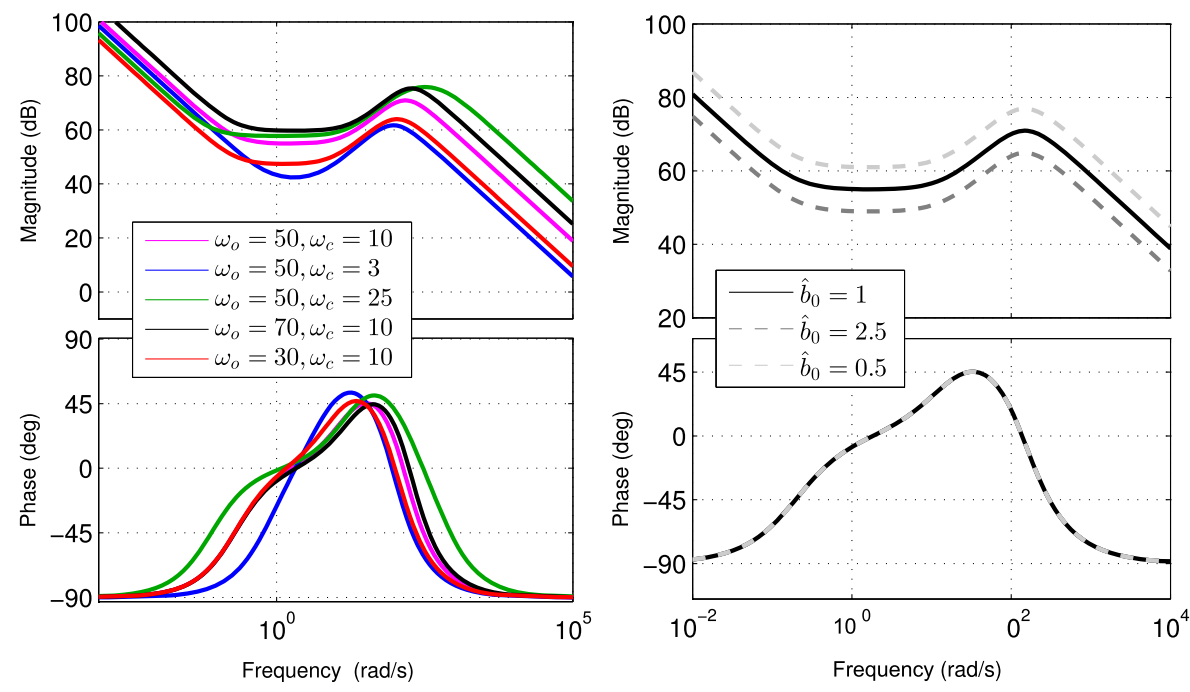

Fig. 3. Frequency-domain analysis of influence of design parameters in error-based ADRC.

Frequency-domain analysis. A loop transfer function of the error-based compact ADRC controller (10) and the considered second order system $G(s)$ can be derived as:

$L_{o}(s)=\frac{b}{\hat{b}_{0}} \cdot \frac{\left(k_{0} l_{1}+k_{1} l_{2}+l_{3}\right) s^{2}+\left(k_{0} l_{2}+k_{1} l_{3}\right) s+k_{0} l_{3}}{\left[s^{2}+\left(l_{1}+k_{1}\right) s+l_{1} k_{1}+l_{2}+k_{0}\right] s}$.

A Bode analysis is conducted for the above system in order to show the influence of design parameters in the error-based compact ADRC. The results are depicted in Fig. 3. It can be noted that the relations of the design parameters on the behavior of the closed-loop system are inherited from the conventional output-based ADRC (as seen in Gao, 2003). Same crucial importance of the input gain $\left(\hat{b}_{0}\right)$ can be spotted, since it scales the entire control action thus significantly influencing the tracking quality, as it evident from (6), (10), and (15).

\subsection{Comments}

Until now, following observations regarding the 1DOF compact error-based form ADRC can be made.

In contrary to the typical output-based design, the error-based ADRC does not rely on the availability of target derivatives $(\dot{r}, \ddot{r}, \ldots)$ for controller synthesis, which has beneficial practical implications. Target derivatives would have to be otherwise measured, calculated (if reference $r$ is known analytically in advance), or reconstructed in real-time with a differentiator based on the known reference signal $(r)$. Each one is problematic in engineering practice and the associated problems complicate further if one considers governing high-order systems and/or significantly noisy measurements. In the error-driven ADRC, these signals are conveniently estimated by the ESO (as part of the total disturbance).

The error-driven ADRC compact form combines the advantages of two types of designs, as it is illustratively depicted in Fig. 4. One is the 


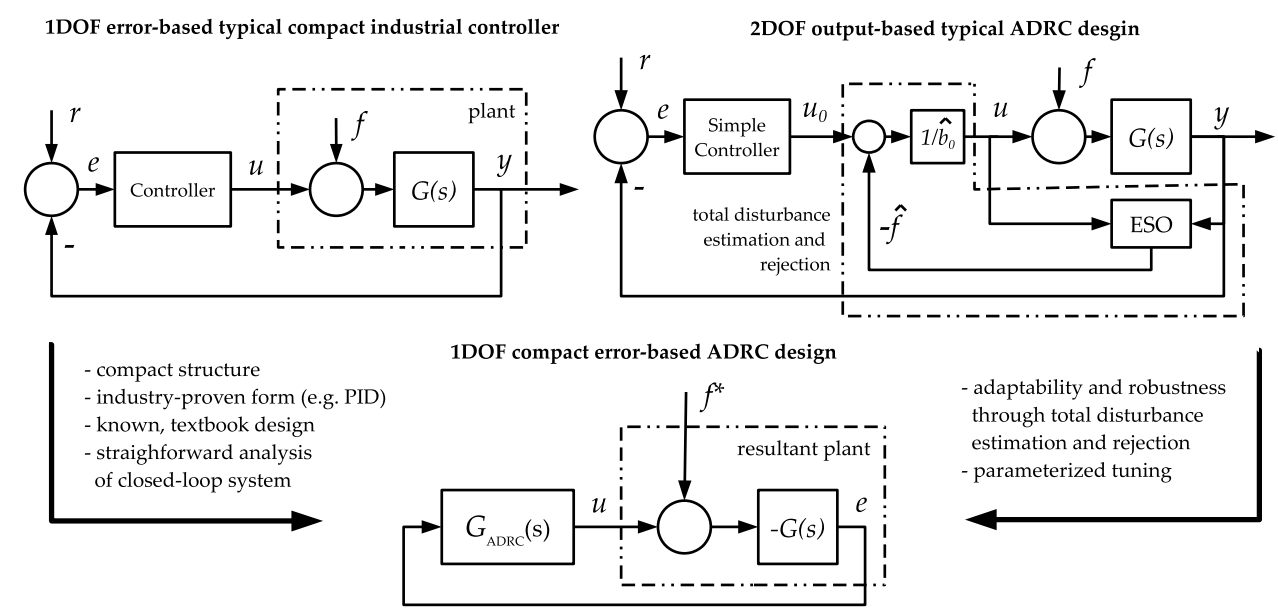

Fig. 4. Illustrative justification of using 1DOF error-based compact ADRC design.

typical 1DOF industrial error-driven design (popularized by PID) and the other is the 2DOF output-based ADRC (with two distinctive loops for disturbance rejection and resultant system control). It should be noted, however, that a compromise is made here between flexibility (offered by the output-based design, in which typical elements like the profile generator, observer, and controller can be designed, tuned, and tested separately) and simplicity (offered by the error-based design, in which the entire ADRC is encapsulated in a single block). As this work focuses on potential deployments in industrial settings, the latter is of greater interest here.

\section{Error-based ADRC design: general case}

\subsection{Plant modeling}

Based on the previous examples of the error-based ADRC, this methodology is generalized here and a class of systems for which it can be applied to is established. The derivation is based on a slightly modified version of a generic $n$th order SISO system model from Michalek (2016). It uses both time-domain and s-domain, which is convenient in this work as the goal is to derive a general single transfer function ADRC solution ${ }^{5}$ :

$\Psi^{(n)}: A(s)[y]+\bar{f}\left(t, y, \dot{y}, \ddot{y}, y^{(3)}, \ldots, y^{(n-1)}, p\right)=B(s)[u]+w$,

where coprime polynomials $A(s)=s^{n}+a_{n-1} s^{n-1}+\cdots+a_{1} s+a_{0}$ and $B(s)=b_{m} s^{m}+b_{m-1} s^{m-1}+\cdots+b_{1} s+b_{0}$, both with uncertain parameters, constitute the linear part of the system model, $\bar{f}(\cdot)$ with its uncertain structure and parameters represents the combination of nonlinear parts of the plant dynamics and overall parametric mismatch ( $p$ ), whereas $w$ describes collectively the acting external interferences.

Following assumptions are made on the above plant model, which are not difficult to satisfy in practice.

A4. $G(s) \triangleq \frac{B(s)}{A(s)}$ is proper.

A5. Roots of $B(s)=0$ belong to the open $\left(b_{0} \neq 0\right)$ left-half complex plane.

A6. Both degree $n$ of polynomial $A(s)$ and sign of $b_{0}$ are known.

A7. Total disturbance is time-differentiable with first time-derivative bounded.

5 Following the source material (Michalek, 2016), notation $v=H(s)[x]$ means that time-dependent signal $v(t)$ is a result of filtration of time-dependent signal $x(t)$ with a filter described with a transfer function $H(s)$.

\subsection{Expressing control task in error-based form}

Here again, instead of following a conventional approach seen in output-based ADRC design based on defining the resultant disturbance as:

$d \triangleq \frac{1}{B(s)}[w-\bar{f}(\cdot)]$,

which then allows to express the system (16) as:

$$
\begin{aligned}
\Psi^{(n)}: y & =G(s)[u+d] \\
\stackrel{\mathrm{A} 6}{\Longrightarrow} y^{(n)} & =\underbrace{-\sum_{j=0}^{n-1} a_{j} y^{(j)}+\sum_{i=0}^{m} b_{i}\left(u^{(i)}+d^{(i)}\right)-0, \ldots, m}_{f\left(y^{(j)}, u^{(i)}, d^{(i)}, p\right) \text { for }}
\end{aligned}
$$

here, the utilized control modification deals with expressing the trajectory-following task in error-based form.

Hence, by recalling the tracking error $e(t)$, one can substitute it to the left-hand side of (18), which results in closed-loop error equation:

$$
\begin{aligned}
e & \triangleq \\
& \stackrel{(18)}{=} r-G(s)[u+d] \\
& =-G(s)\left[u+d-\frac{r}{G(s)}\right] \\
& =-G(s)\left[u+d-u_{r}\right] \\
& =-G(s)[u+\tilde{d}],
\end{aligned}
$$

where $u_{r}=A(s)[r] / B(s)=[r] / G(s)$ is the perturbing control action caused by the reference trajectory $r$ and $\tilde{d} \triangleq d-u_{r}$ is the new resultant external perturbation affecting the system. Rewriting (19) in time domain gives:

$\Sigma^{(n)}: e^{(n)}+\sum_{j=0}^{n-1} a_{j} e^{(j)}=-\sum_{j=1}^{m} b_{j} u^{(j)}-\sum_{j=0}^{m} b_{j} \tilde{d}^{(j)}-b_{0} u$.

Similar to (35), the parametric mismatch related to the input gain can be treated as part of the disturbance, which reduces the error dynamics to:

$$
\Sigma^{(n)}: \quad e^{(n)}=\underbrace{-\sum_{j=0}^{n-1} a_{j} e^{(j)}-\sum_{j=1}^{m} b_{j} u^{(j)}-\sum_{j=0}^{m} b_{j} \tilde{d}^{(j)}-\left(b_{0}-\hat{b}_{0}\right)}_{\tilde{f}\left(e^{(i)}, u^{(j)}, \tilde{d}^{(j)}, p\right) \text { for } i=0, \ldots, n-1, j=0, \ldots, m} u-\hat{b}_{0} u,
$$

where $\tilde{f}(\cdot)$ is the lumped disturbance, which now also includes the effect of uncertain input gain. 
Table 2

General forms of controller gains for $n$th order plant model parametrized by frequency $\omega_{c}$ (here for $n=\{1,2,3,4\}$ ).

\begin{tabular}{llllll}
\hline$n$ & $\cdots$ & $k_{3}$ & $k_{2}$ & $k_{1}$ & $k_{0}$ \\
\hline 1 & $\cdots$ & - & - & - & $\omega_{c}$ \\
2 & $\cdots$ & - & - & $2 \omega_{c}$ & $\omega_{c}^{2}$ \\
3 & $\cdots$ & - & $3 \omega_{c}$ & $3 \omega_{c}^{2}$ & $\omega_{c}^{3}$ \\
4 & $\cdots$ & $4 \omega_{c}$ & $6 \omega_{c}^{2}$ & $4 \omega_{c}^{3}$ & $\omega_{c}^{4}$ \\
$\vdots$ & $\therefore$ & $\vdots$ & $\vdots$ & $\vdots$ & $\vdots$ \\
\hline
\end{tabular}

\subsection{Control rule design and tuning}

As it was shown in Section 2.2, the desired error dynamics for the closed-loop system can be shaped by bilaterally adding control term $\sum_{i=1}^{n-1} k_{i} e^{(i)}$ to (21), resulting in:

$$
\bar{\Sigma}^{(n)}: e^{(n)}+\sum_{i=1}^{n-1} k_{i} e^{(i)}=\underbrace{\sum_{i=1}^{n-1} k_{i} e^{(i)}+\tilde{f}\left(e^{(i)}, u^{(j)}, \tilde{d}^{(j)}, p\right)}_{f^{*}\left(e^{(i)}, u^{(j)}, \tilde{d}^{(j)}, p\right) \text { for } i=0, \ldots, n-1, j=0, \ldots, m}-\hat{b}_{0} u,
$$

where $k_{i}>0$ are design control coefficients (to be selected) and $f^{*}(\cdot)$ is the total disturbance in the error-based representation (cf.(18)), to be reconstructed and then timely rejected by the control input $(u)$.

Now, assuming temporary that the resultant disturbance is accurately reconstructed with the ESO (i.e. $\hat{f}^{*}=f^{*}$ ) and that $\hat{b}_{0}=b_{0}$, one can propose a following governing action:

$u\left(e, \hat{f}^{*}\right)=\frac{1}{\hat{b}_{0}}\left(u_{0}(e)+\hat{f}^{*}\right)$,

where $u_{0} \triangleq k_{0} e$ is arbitrary chosen to be just a simple proportional output-feedback controller. By applying control action (23) to the dynamics (22), one obtains the following closed-loop error dynamics:

$e^{(n)}+k_{n-1} e^{(n-1)}+\cdots+k_{1} \dot{e}+k_{0} e=0$,

which determines the prescribed control behavior of the resultant (theoretically) disturbance-free chain of integrators, in accordance to gains $k_{i}>0$, for $i=0, \ldots, n-1$.

Remark 3. One can notice the crucial importance of the ESO in timely and accurately estimating uncertain term $f^{*}$. Compared to the typical output-based ADRC, its reconstruction is additionally burdened here with the uncertainty $u_{r}$, being the result of inclusion of unknown reference time-derivatives effect. This is the consequence of obtained major simplification related to reduced controller form and lack of necessity for a differentiator.

Same popular and effective parametric synthesis of the controller, based on pole-placement approach (Gao, 2003) and known from the conventional ADRC tuning, can be used for the considered compact error-based version. It is based on defining a frequency $\omega_{c}>0$, which determines the desired bandwidth $\Omega_{c}=\left[0, \omega_{c}\right]$ of the closed-loop system. Comparison of the characteristic polynomial $P_{c}(s)$ of dynamics (24) with a desired polynomial $P_{c}^{*}(s) \triangleq\left(s+\omega_{c}\right)^{n}$ :

$$
\begin{aligned}
P_{c}(s) & :=P_{c}^{*}(s), \\
s^{(n)}+k_{n-1} s^{(n-1)}+\cdots+k_{1} s+k_{0} & :=\left(s+\omega_{c}\right)^{n},
\end{aligned}
$$

which places all the poles at one location $-\omega_{c}$, greatly simplifies the tuning process, as it is reduced to a single parameter selection $\left(\omega_{c}\right)$ for controller coefficients computation. Some general forms of the controller gains for the $n$th order plant model parametrized by $\omega_{c}$ are gathered in Table 2.

\subsection{Disturbance observer design and tuning}

Now, for system (22), a following set of state variables can be chosen:

$x_{1} \triangleq e, \quad x_{2} \triangleq \dot{e}, \quad \ldots, \quad x_{n} \triangleq e^{(n-1)}, \quad x_{n+1} \triangleq f^{*}$,

thus resulting in a $(n+1)$ th order extended state vector $\boldsymbol{x}^{\top}=\left[\begin{array}{lll}x_{1} & x_{2} & \ldots\end{array}\right.$ $\left.x_{n+1}\right]$ and extended disturbance vector $\boldsymbol{F}^{\top}=\left[\begin{array}{llll}0 & \ldots & 0 & x_{n+1}\end{array}\right]$, in which the extra virtual state variable represents the total disturbance. The system model (22) in state-space can be now described in extended form as:

$\bar{\Sigma}^{(n+1)}:\left\{\begin{array}{l}\dot{x}=\boldsymbol{A} \boldsymbol{x}+\boldsymbol{b} u+\boldsymbol{h} \dot{\boldsymbol{F}}, \\ e=\boldsymbol{c}^{\top} \boldsymbol{x},\end{array}\right.$

$\boldsymbol{A}=\left[\begin{array}{cccccc}0 & 1 & 0 & \cdots & 0 & 0 \\ 0 & 0 & 1 & \cdots & 0 & 0 \\ 0 & 0 & 0 & \cdots & 0 & 0 \\ \vdots & \vdots & \vdots & \ddots & \vdots & \vdots \\ 0 & -k_{1} & -k_{2} & \cdots & -k_{n-1} & 1 \\ 0 & 0 & 0 & \cdots & 0 & 0\end{array}\right], \boldsymbol{b}=\left[\begin{array}{c}0 \\ 0 \\ \vdots \\ 0 \\ -\hat{b}_{0} \\ 0\end{array}\right], \boldsymbol{h}=\left[\begin{array}{c}0 \\ 0 \\ 0 \\ \vdots \\ 0 \\ 1\end{array}\right], \boldsymbol{c}=\left[\begin{array}{c}1 \\ 0 \\ 0 \\ 0 \\ 0 \\ 0\end{array}\right]$.

Since the system (27) is observable, one can propose a following $(n+1)$ th order ESO to estimate its states, which only uses practically available plant input and output signals:

$\hat{\bar{\Sigma}}^{(n+1)}:\left\{\begin{array}{l}\dot{z}=\boldsymbol{A z}+\boldsymbol{b} u+l\left(x_{1}-\boldsymbol{c}^{T} \hat{\boldsymbol{x}}\right), \\ \hat{e}=\boldsymbol{c}^{\top} \boldsymbol{z},\end{array}\right.$

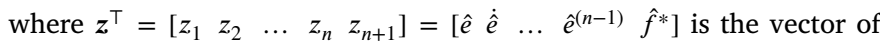
state variables estimates, and $\boldsymbol{l}^{\top}=\left[\begin{array}{llllll}l_{1} & l_{2} & \ldots & l_{n} & l_{n+1}\end{array}\right]$ is the vector of observer gains (to be selected).

It is notable that the prescribed error dynamics (24) is incorporated into the ESO structure (with the exception of $k_{0}$ ), making the state matrix $\boldsymbol{A}$ dependent on the design coefficients $k_{0}, \ldots, k_{n-1}$. This trivializes the outer-loop controller as it is reduced in (23) to just a proportional output-error feedback action, regardless of the order of the system dynamics. That feature is especially appealing to practitioners as it simplifies the control, compared to conventional ADRC design, where the outer loop requires the availability of the reference time-derivatives up to the order of dynamics (Michalek, 2016; Zhang, 2017).

The pole-placement technique for observer gains parametrization, known from the conventional ADRC design, can be used for the errorbased form as well. The goal is to place the roots of characteristic polynomial $P_{o}(\lambda)$ in the complex plane (desirably far) on the left-hand side of the roots determined in Section 3.3. Following previous results related to ESO tuning (Gao, 2003), it can be effectively done by forcing:

$$
\begin{aligned}
P_{o}(\lambda) & :=P_{o}^{*}(\lambda), \\
\operatorname{det}(\lambda \boldsymbol{I}-\boldsymbol{H}) & :=\left(\lambda+\omega_{o}\right)^{n+1},
\end{aligned}
$$

where $\omega_{o}$ is a design frequency that determines the observer bandwidth $\Omega_{o}=\left[0, \omega_{o}\right]$ and the estimation-error state matrix has the form:

$\boldsymbol{H}=\boldsymbol{A}-\boldsymbol{l} \boldsymbol{c}^{\top}=\left[\begin{array}{cccccc}-l_{1} & 1 & 0 & \cdots & 0 & 0 \\ -l_{2} & 0 & 1 & \cdots & 0 & 0 \\ -l_{3} & 0 & 0 & \cdots & 0 & 0 \\ \vdots & \vdots & \vdots & \ddots & \vdots & \vdots \\ -l_{n} & -k_{1} & -k_{2} & \cdots & -k_{n-1} & 1 \\ -l_{n+1} & 0 & 0 & \cdots & 0 & 0\end{array}\right]$.

Some general forms of the observer gains for the $(n+1)$ th order plant model parametrized by frequency $\omega_{o}$ are gathered in Table 3 .

Finally, the derivation of a general form of the considered 1DOF error-driven ADRC solution is based on combining the general governing action (23) with the general observer form (28), following the procedure seen in the example in Section 2. Expressing this combination in transfer 
Table 3

General forms of observer gains for $n$th order plant model parametrized by frequency $\omega_{o}$ (here for $n=\{1,2,3\}$ ).

\begin{tabular}{llllll}
\hline$n$ & $\cdots$ & $l_{4}$ & $l_{3}$ & $l_{2}$ & $l_{1}$ \\
\hline 1 & $\cdots$ & - & - & $\omega_{o}^{2}$ & $2 \omega_{o}$ \\
2 & $\cdots$ & - & $\omega_{o}^{3}$ & $3 \omega_{o}^{2}-l_{1} k_{1}$ & $3 \omega_{o}-k_{1}$ \\
3 & $\cdots$ & $\omega_{o}^{4}$ & $4 \omega_{o}^{3}-l_{1} k_{1}-l_{2} k_{2}$ & $6 \omega_{o}^{2}-k_{1}-l_{1} k_{2}$ & $4 \omega_{o}-k_{2}$ \\
& $\vdots$ & $\vdots$ & $\vdots$ & $\vdots$ & $\vdots$ \\
\hline
\end{tabular}

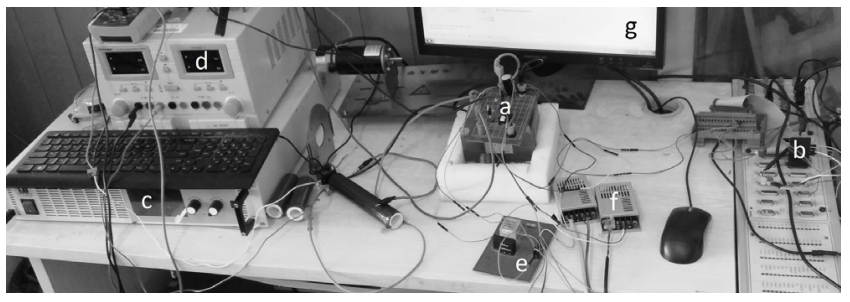

Fig. 5. Laboratory setup used in the experiments, with a - buck converter circuit, b dSpace DS 1103 real-time controller, c - DC input voltage, d - digital oscilloscope, e voltage and currents sensors, f - A/D converters, and g - PC-based dSpace ControlDesk software.

function yields ${ }^{6}$ :

$$
\begin{aligned}
U(s) & =\frac{1}{\hat{b}_{0}} \cdot \frac{L(s)}{M(s)} \cdot \overbrace{[R(s)-Y(s)]}^{E(S)}, \\
G_{\mathrm{ADRC}}(s) & =\frac{U(s)}{E(s)}=\frac{1}{\hat{b}_{0}} \cdot \frac{L(s)}{M(s)},
\end{aligned}
$$

for:

$M(s)=\sum_{i=0}^{n}\left(\sum_{q=0}^{i} \kappa_{q} \beta_{i-q}\right) s^{n+1-i}, \quad L(s)=\sum_{i=0}^{n}\left(\sum_{q=0}^{n-i} \kappa_{i+q} \beta_{n+i-q}\right) s^{n-i}$,

where $\beta_{j}=l_{j}$ for $j=1,2, \ldots, n+1$ and $\kappa_{n-i}=k_{i}$ for $i=0,1, \ldots, n-1$ strictly corresponds to the design parameters of the outer-loop controller and the observer, which can be straightforwardly computed based on Tables 2 and 3. A rigorous mathematical proof of the considered errorbased control design is given in the Appendix.

\section{Simulation and experimental validation}

\subsection{Preliminaries}

In order to verify the compact error-based ADRC, a problem of fast and precise output voltage tracking in a highly uncertain DC-DC buck power converter is considered. The utilized rapid prototyping platform is depicted in Fig. 5. A combination of software-in-the-loop (SIL) and hardware-in-the-loop (HIL) experiments is performed using dSPACE DS 1103 real-time controller. The implementation is realized in Matlab/Simulink environment, while communication is established using a dedicated real-time toolbox with sampling period $T_{s}=200$ us.

Based on Sira-Ramirez and Silva-Ortigoza (2006), a following average model of the considered converter is used in the conducted tests:

$\Psi^{(2)}:\left\{\begin{array}{l}\frac{d v_{o}(t)}{d t}=\frac{1}{C} i_{L}(t)-\frac{1}{C R} v_{o}(t), \\ \frac{d i_{L}(t)}{d t}=\frac{V_{\text {in }}}{L} u(t)-\frac{1}{L} v_{o}(t),\end{array}\right.$

where $u \in[0,1]$ is the duty ratio (control signal), $v_{o}[\mathrm{~V}]$ is the average capacitor output voltage (controlled signal), $i_{L}[\mathrm{~A}]$ is the average inductor current, $R[\Omega]$ is the load resistance of the circuit, $L[\mathrm{H}]$ is the

6 The formula happens to correspond to the system used for ADRC frequency analysis in Xue and Huang (2015). filter inductance, $C[\mathrm{~F}]$ is the filter capacitance, and $V_{\text {in }}[\mathrm{V}]$ is the input DC voltage source. The structure of the above plant model is known, but its parameters remain uncertain (and potentially time-varying). The control objective is to make the average output voltage signal $\left(v_{o}\right)$ track a given smooth trajectory $\left(v_{r}\right)$ by manipulating the duty ratio $(u)$, even in the presence of plant parametric uncertainties and unmodeled external disturbances.

Based on the expert knowledge about the used power circuit, it is possible to a'priori obtain rough parameters of the plant mathematical model, namely: $V_{\text {in } 0}=20 \mathrm{~V}, L_{0}=0.01 \mathrm{H}, C_{0}=0.001 \mathrm{~F}$, and $R_{0}=50 \Omega$, which are used as nominal values in the upcoming tests. An open-loop comparison between a real system and its utilized analytical description in Fig. 6 shows that the plant model roughly captures main behavior of the actual setting.

\subsection{Control scheme implementation}

Following the standard ADRC line of reasoning, the plant model from is first reformulated, emphasizing the system causal input-output relation:

$\Psi^{(2)}: \frac{d^{2} v_{o}}{d^{2} t}=\underbrace{-\frac{1}{C R}}_{d_{1}} \frac{d v_{o}}{d t} \underbrace{-\frac{1}{C L}}_{d_{2}} v_{o}+\underbrace{\frac{V_{\mathrm{in}}}{C L}}_{b_{0}} u+w$,

where $w$ is additionally introduced to model the unknown (possibly time-varying and nonlinear) matched external perturbation. Combining all the uncertain (or unknown) terms of the above model, including the uncertainty in modeling the input gain, results in a following compact form:

$\Psi^{(2)}: \ddot{v}_{o}=\underbrace{d_{1} v_{o}+d_{2} \dot{v}_{o}+b_{0} u-\hat{b}_{0} u+w}_{f\left(t, \dot{v}_{o}, v_{o}, u, p, w\right)}+\hat{b}_{0} u=f+\hat{b}_{0} u$.

Now, it can be straightforwardly verified that the considered dynamics of the buck converter (34) belongs to the class of systems defined by (16), with $A(s)=d_{1} s+d_{2}$ and $B(s) \equiv b_{0}$. For the same plant model, after transforming the control task into error-based form, as shown in (17) through (22), one gets the error dynamics of buck converter system:

$e \triangleq v_{r}-v_{o} \Rightarrow \dot{e} \triangleq \dot{v}_{r}-\dot{v}_{o} \Rightarrow \Sigma^{(2)}: \ddot{e} \stackrel{(35)}{=} \underbrace{\ddot{v}_{r}-f}_{f^{*}}-\hat{b}_{0} u$.

For such second order dynamics, it is clear that the ADRC controller in form of (10) can be straightforwardly used as:

$$
G_{\mathrm{ADRC}}(s)=\underbrace{\frac{U(s)}{V_{r}(s)-V_{o}(s)}}_{E(s)}=\frac{\left(k_{0} l_{1}+k_{1} l_{2}+l_{3}\right) s^{2}+\left(k_{0} l_{2}+k_{1} l_{3}\right) s+k_{0} l_{3}}{\hat{b}_{0}\left[s^{2}+\left(l_{1}+k_{1}\right) s+l_{1} k_{1}+l_{2}+k_{0}\right] s},
$$

or in discrete form (using Tustin approximation) as:

$G_{\mathrm{ADRC}}(z)=\frac{U(z)}{E(z)}=\frac{T_{s}}{2 \hat{b}_{0}} \frac{\left(L_{3} z^{3}+L_{2} z^{2}+L_{1} z+L_{0}\right)}{\left(M_{2} z^{2}+M_{1} z+M_{0}\right)(z-1)}$,

with polynomial coefficients defined as:

$L_{3}=4 l_{3}+4 l_{1} k_{0}+2 T_{s} k_{0} l_{2}+2 T_{s} l_{3} k_{1}+T_{s}^{2} l_{3} k_{0}+4 k_{1} l_{2}$,

$L_{2}=-4 l_{3}-4 k_{1} l_{2}+2 k_{0} l_{2} T_{s}+3 k_{0} l_{3} T_{s}^{2}-4 k_{0} l_{1}+2 k_{1} l_{3} T_{s}$,

$L_{1}=-2 T_{s} l_{3} k_{1}-4 l_{3}-4 k_{1} l_{2}-2 k_{0} l_{2} T_{s}+3 k_{0} k_{3} T_{s}^{2}-4 k_{0} l_{1}$,

$L_{0}=4 l_{3}+4 k_{1} l_{2}+4 l_{1} k_{0}+k_{0} l_{3} T_{s}^{2}-2 k_{1} l_{3} T_{s}-2 k_{0} l_{2} T_{s}$,

$M_{2}=2 l_{1} T_{s}+2 k_{1} T_{s}+k_{0} T_{s}^{2}+l_{2} T_{s}^{2}+k_{1} l_{1} T_{s}^{2}+4$,

$M_{1}=2 l_{2} T_{s}^{2}+2 l_{1} k_{1} T_{s}^{2}+2 k_{0} T_{s}^{2}-8$,

$M_{0}=-2 k_{1} T_{s}+k_{1} l_{1} T_{s}^{2}+k_{0} T_{s}^{2}+l_{2} T_{s}^{2}-2 l_{1} T_{s}+4$.

The observer and controller gains are again selected directly according to Tables 2 and 3, which makes the entire considered control strategy 


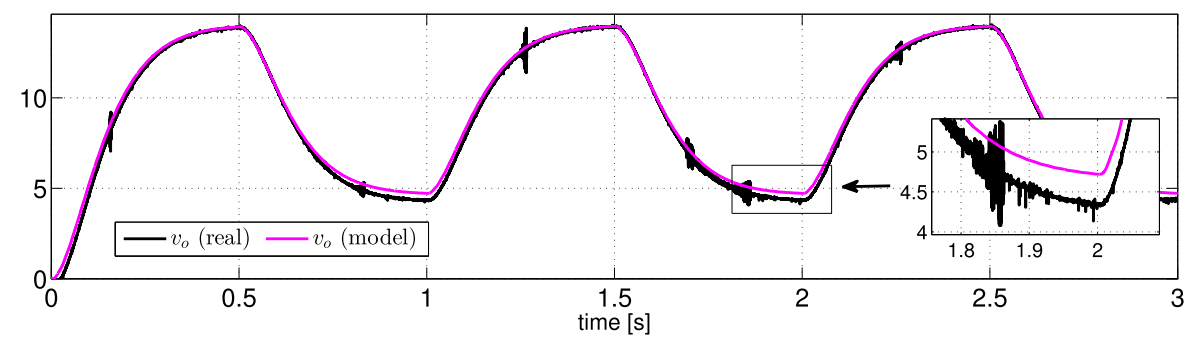

Fig. 6. Comparison between the real DC-DC buck power converter and its obtained model.

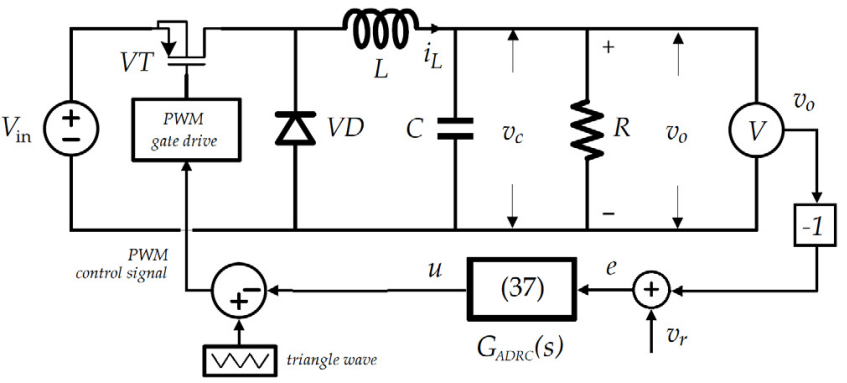

Fig. 7. Proposed 1DOF compact error-based ADRC for the DC-DC buck converter.

tuned only by three intuitive design parameters $\left(\omega_{o}, \omega_{c}\right.$, and $\left.\hat{b}_{0}\right)$. The application of the compact error-based ADRC for the considered buck converter is illustratively depicted in Fig. 7.

\subsection{Conducted tests and obtained results}

Three types of SIL tests are performed (SIL1-SIL3). First one (SIL1) verifies the nominal performance of the error-based ADRC for the buck converter system in terms of accuracy of smooth trajectory tracking (being a square signal filtered by a stable second-order order dynamics), energy consumption, and observer estimation precision. The system is simulated with band-limited white measurement noise with power $\sigma=10^{-11}$. Beyond this point, no re-tuning is done. In the next test (SIL2), robustness of the closed system is investigated by emulating parameters variations $\left(R \neq R_{0}, V_{\text {in }} \neq V_{\text {ino } 0}\right)$ as well as by checking the performance recovery under various types of input disturbances (constant, sawtooth, and harmonic). A quantitative comparison with a conventional ADRC design scheme is conducted in SIL3 for a reference signal with increased amplitude. Finally, the performance of the errorbased design is validated in HIL1. In all of the conducted runs and considered control algorithms, same tuning parameters have been chosen and kept throughout the tests (with no retuning), namely: $\hat{b}_{0}=20000$, $\omega_{c}=130$, and $\omega_{o}=50 \omega_{c}$. The parameters were chosen empirically, following guidelines in Gao (2003), with a compromise between speed of estimation/tracking and noise amplification.

The results of SIM1 are gathered in Fig. 8. One can notice that the trajectory is tracked with an acceptable accuracy $(|e|<0.15)$, while the control signal does not reach the saturation boundaries $(0 \leq u<0.32)$. The estimated total disturbance $\left(\hat{f}^{*}\right)$ is compared here with a roughly approximated total disturbance: $f^{*} \approx \ddot{e}+\hat{b}_{0} u$ (cf.(36)) resulting in a difference within close vicinity of zero $\left(\left|\epsilon_{f}\right|<0.1\right)$. This, together with the achieved level of estimation error $\left(|\epsilon|<5 \cdot 10^{-3}\right)$ can be an indication that the total disturbance is being timely and precisely reconstructed by the ESO. The outcomes of SIM2 are collectively shown in Fig. 9. Even in the presence of external disturbance of various types, the tracking accuracy is kept within practically satisfactory level $(|e|<2.5)$. In case of parametric robustness, the change of resistance $(R)$ did not have much effect on the quality of closed-loop system. Variations in the input voltage $\left(V_{\text {in }}\right)$ did however influence the control results visibly, but for this particular plant such changes are still practically acceptable. The results of SIM3 are presented in Fig. 10. First, the compact error-driven ADRC was compared with the conventional output-based ADRC with FF in terms of tracking accuracy. No significant differences can be noted, but it has to be underlined that the proposed compact version does not require the information about the derivatives of the target signal, which is a huge practical advantage. In case of output-based ADRC with FF, two TDs had to be additionally implemented and tuned, which complicated the overall control deployment. Lastly, the results of HIL1 are gathered in Fig. 11. The trajectory is tracked with an acceptable accuracy $(|e|<0.2)$, while the control signal does not exceed permissible level $(0 \leq u<0.9)$. The estimation part is also satisfactory, despite the presence of measurement noise from voltage sensor $(|\epsilon|<0.015)$.

\section{Conclusions}

In this work, a 1DOF error-based ADRC formulation has been systematically analyzed. Such design allows the standard ADRC algorithm (with all its beneficial features) to be realized in a compact, almost plug\&play form, commonly found in industrial control configurations. With the ADRC expressed in error-based form, its typical components, which were previously working separately, can now be bundled into a single structure, easily implementable in industrial automation software. This potentially increases the competitiveness of ADRC against the ubiquitous PID-like controllers. The considered approach, in its general form, has been rigorously proved here by means of singular perturbation theory. Additionally, experimental tests using a power electronics device have been successfully conducted to further validate the design.

\section{Acknowledgments}

This work was supported in part by the Fundamental Research Funds for the Central Universities under Grant 3208008602 and Southeast University postdoctoral fund under Grant 1108000201 . This work was also supported in part by the National Natural Science Foundation of China under Grant 61573099, Grant 61633003, and Grant 61750110525. The authors would like to thank Lu Zhang and Han Wu for setting up the laboratory platform.

\section{Appendix. Stability analysis of general error-based ADRC}

The theoretical investigation of the generalized version of the compact error-based ADRC is presented next and its analysis is based on singular perturbation theory (Kokotovic, Khalil, \& O'Reilly, 1999; Shao \& Gao, 2017). Compared to Shao and Gao (2017), here the stability proof will not be divided explicitly into slow and fast dynamics representing the controller and observer loops, respectively. In the considered modified ADRC these two loops are incorporated into a single closed-loop error dynamics. For the same reason, the analysis below also contains (implicitly) the convergence of the estimation error.

First, the general expression given in (28) can be expressed alternatively as:

$\dot{\boldsymbol{q}}=\left(\boldsymbol{A}-\boldsymbol{l c} \boldsymbol{c}^{\top}\right) \boldsymbol{q}+\boldsymbol{h} \eta, \quad \eta=\dot{f}^{*}$, 

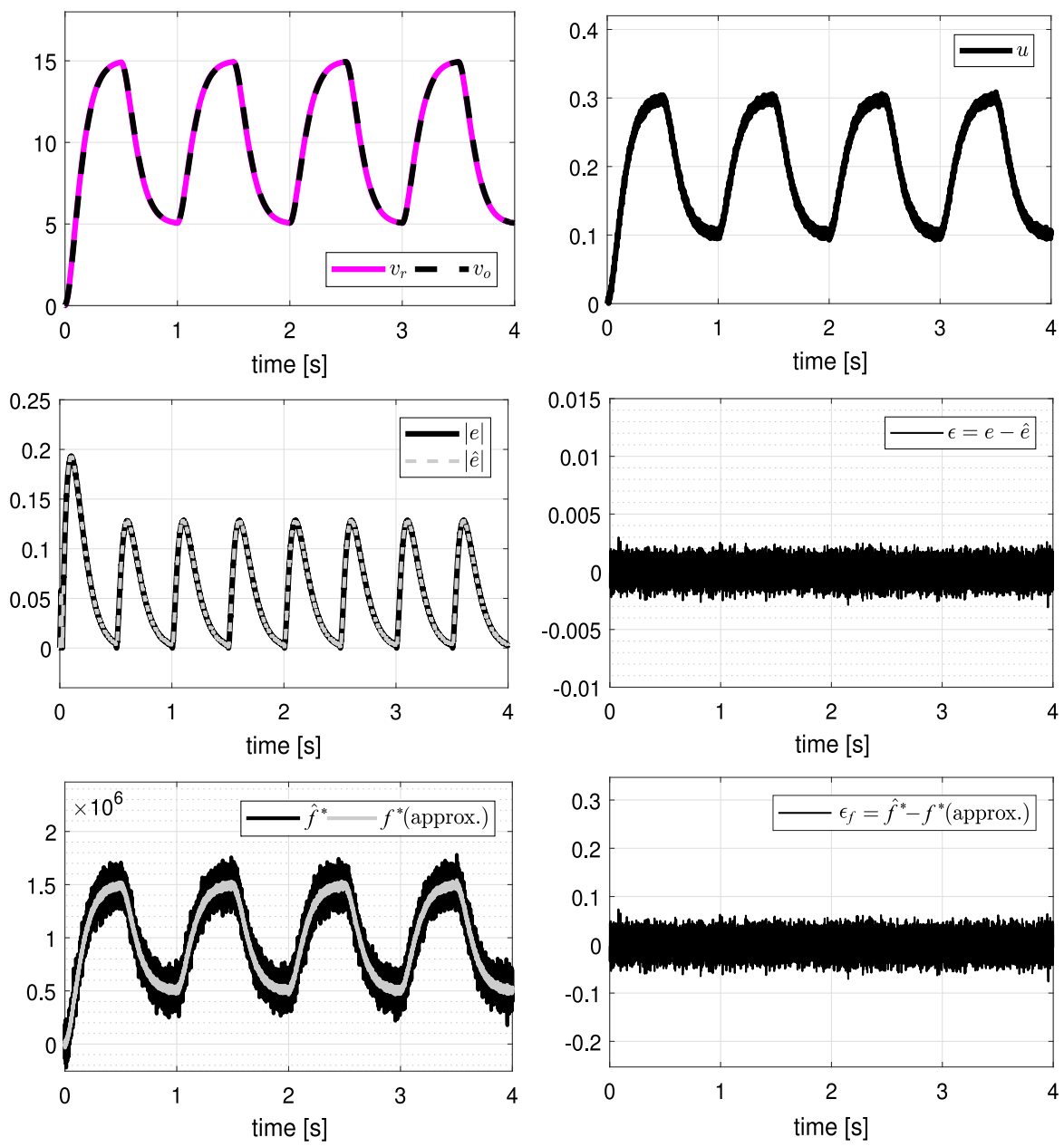

Fig. 8. [SIL1] Nominal performance of the compact error-based ADRC.
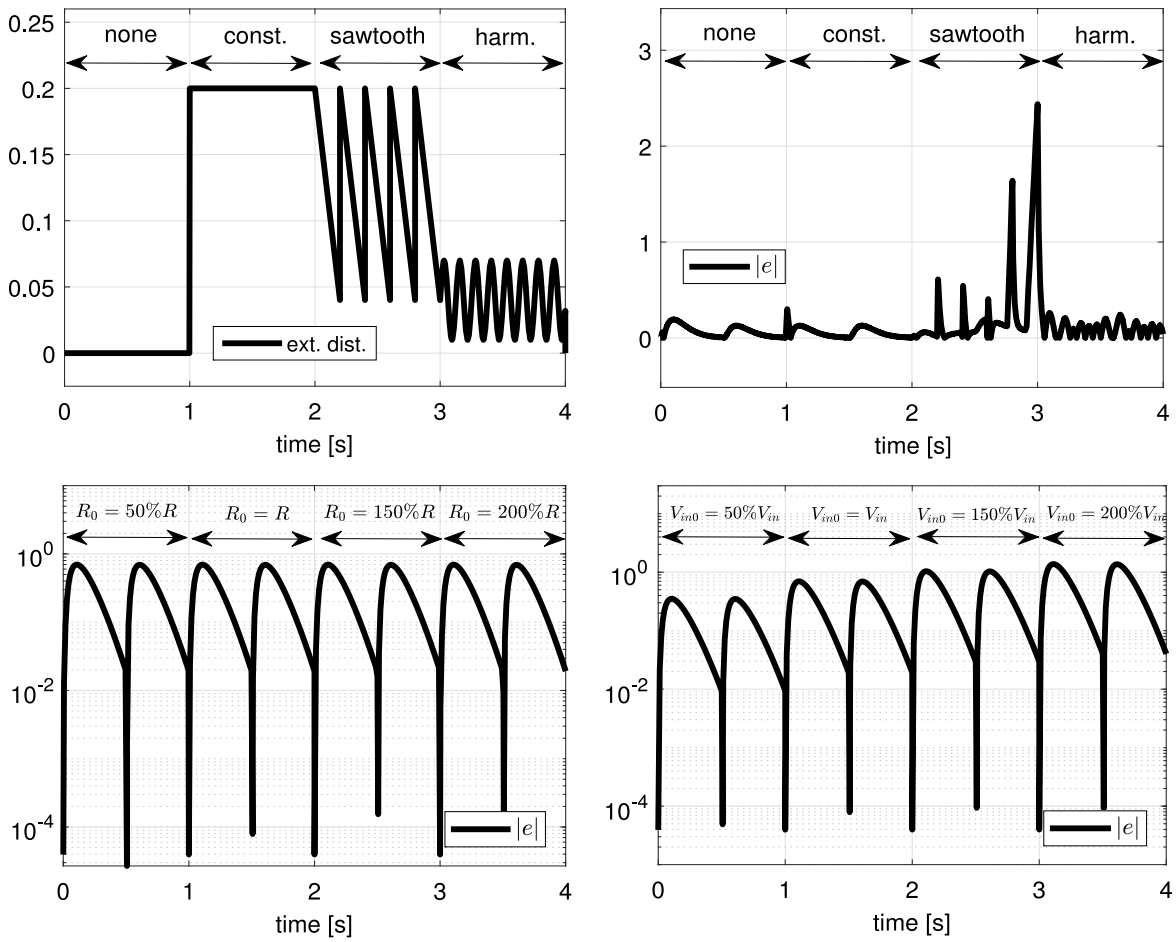

Fig. 9. [SIL2] Robustness tests of the proposed compact error-based ADRC. 

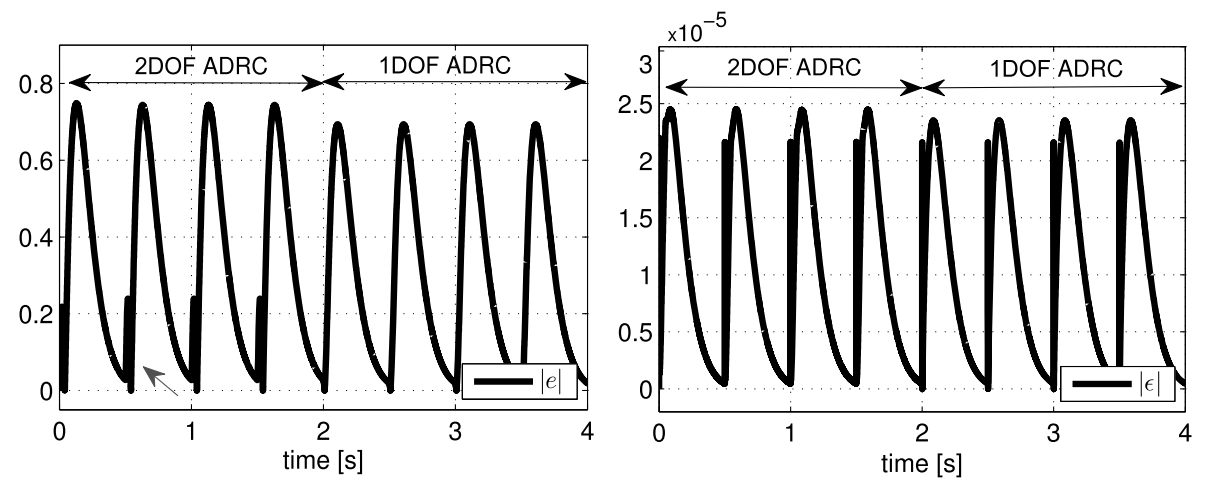

Fig. 10. [SIL3] Quantitative comparison with standard output-based ADRC scheme.
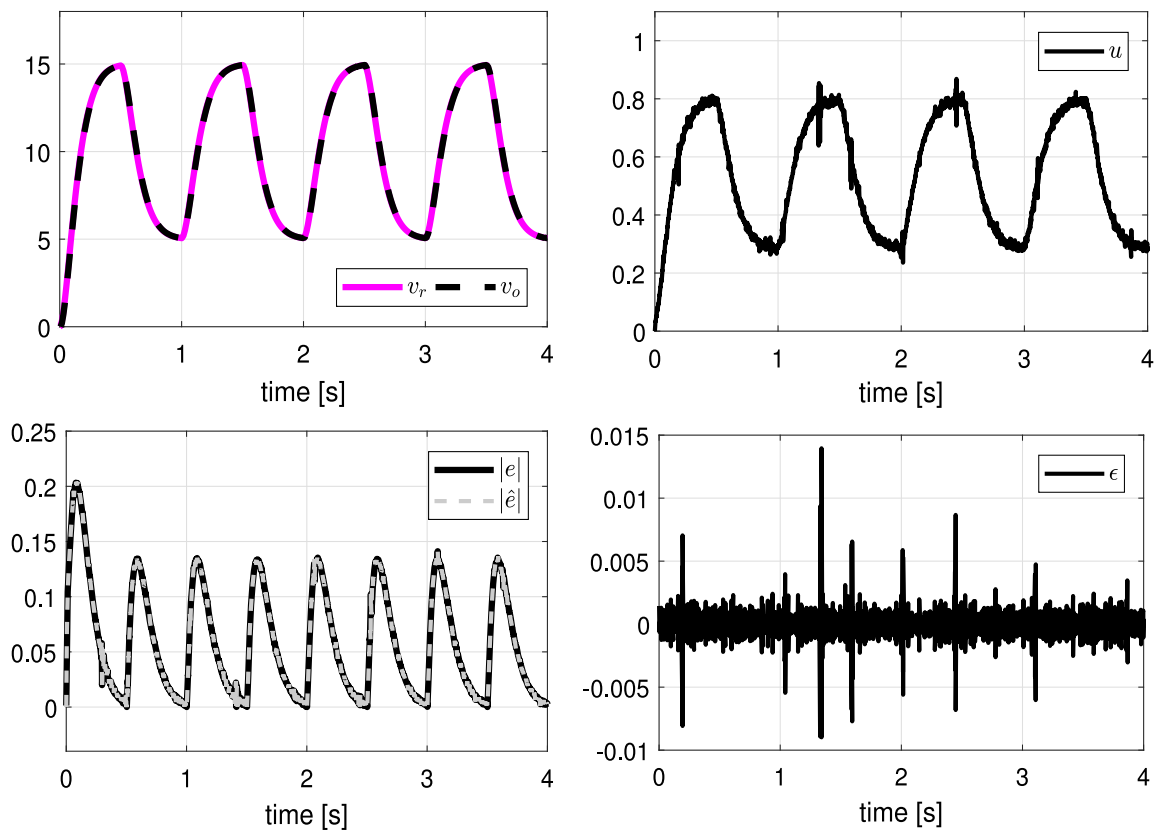

Fig. 11. [HIL1] Nominal performance of the compact error-based ADRC.

by defining $\boldsymbol{q}^{\top}=\left[\begin{array}{llll}q_{1} & \cdots & q_{n} & q_{n+1}\end{array}\right]=\left[\begin{array}{lll}(e-\hat{e}) & \cdots & \left(e^{(n-1)}-\hat{e}^{(n-1)}\right)\end{array}\right.$ $\left.\left(f^{*}-\hat{f}^{*}\right)\right]$.

Next, recalling both the estimation error matrix $\boldsymbol{H}$ from (30) and the pole-placement-based observer tuning from (29), one can induce, by analyzing their forms for different orders ESOs, that in general case:

$\operatorname{det}(\lambda \boldsymbol{I}-\boldsymbol{H})=\lambda^{n+1}+p_{1} \lambda^{n}+p_{2} \lambda^{n-1}+\cdots+p_{n-1} \lambda^{2}+p_{n} \lambda+p_{n+1}$,

$$
=\left(\lambda+\omega_{o}\right)^{n+1} \text {, }
$$

for $\left[p_{1}, p_{2}, \ldots, p_{n}, p_{n+1}\right]=\left[\beta_{1} \omega_{o}, \beta_{2} \omega_{o}^{2}, \ldots, \beta_{n} \omega_{n}, \beta_{n+1} \omega_{o}^{n+1}\right]$. That yields the eigenvalues of the error matrix $\boldsymbol{H}$ :

$\lambda_{m}\{\boldsymbol{H}\}=-\omega_{o}, \quad \forall m=1,2, \ldots, n+1$.

As seen previously, the observer gains and the controller gains are parametrized by the observer bandwidth $\left(\omega_{o}>0\right)$ and the controller bandwidth $\left(\omega_{c}>0\right)$, respectively. In general case, they can be calculated as:

$k_{i}=\frac{n !}{i !(n-i) !} \omega_{c}^{n-i}, \quad 0 \leq i \leq n-1, \quad$ and $\quad \beta_{j}=\frac{(n+1) !}{j !(n+1-j) !}, \quad 1 \leq j \leq n+1$.

The coefficients of (39) can be thus computed as:

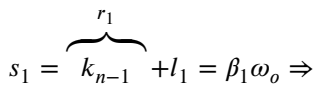

$$
\begin{aligned}
s_{2}= & \overbrace{k_{n-1} l_{1}+k_{n-2}}^{l_{1}=\beta_{1} \omega_{o}-k_{n-1},}+l_{2}=\beta_{2} \omega_{o}^{2} \Rightarrow \\
& \Rightarrow l_{2}=\beta_{2} \omega_{o}^{2}-\left[k_{n-1} \beta_{1} \omega_{o}-\left(k_{n-1}\right)^{2}+k_{n-2}\right],
\end{aligned}
$$

$$
\begin{aligned}
s_{n-1}= & \overbrace{k_{2} l_{1}+k_{3} l_{2}+\cdots+k_{n-1} l_{n-2}+k_{1}}^{r_{n-1}}+l_{n-1}=\beta_{n-1} \omega_{o}^{n-1} \Rightarrow \\
& \Rightarrow l_{n-1}=\beta_{n-1} \omega_{o}^{n-1}-\left[k_{2} l_{1}+k_{3} l_{2}+\cdots+k_{n-1} l_{n-2}+k_{1}\right], \\
s_{n}= & \overbrace{k_{1} l_{1}+k_{2} l_{2}+k_{3} l_{3}+\cdots+k_{n-1} l_{n-1}}^{r_{n}}+l_{n}=\beta_{n} \omega_{o}^{n} \Rightarrow \\
& \Rightarrow l_{n}=\beta_{n} \omega_{o}^{n}-\left[k_{1} l_{1}+k_{2} l_{2}+k_{3} l_{3}+\cdots+k_{n-1} l_{n-1}\right], \\
s_{n+1}= & l_{n+1}=\beta_{n+1} \omega_{o}^{n+1}=\omega_{o}^{n+1},
\end{aligned}
$$

which allows the estimation error state matrix (30) to be rewritten as:

$$
\boldsymbol{H} \stackrel{(43)}{=}\left[\begin{array}{cccccc}
-\beta_{1} \omega_{o}+r_{1} & 1 & 0 & \cdots & 0 & 0 \\
-\beta_{2} \omega_{o}^{2}+r_{2} & 0 & 1 & \cdots & 0 & 0 \\
\vdots & 0 & 0 & \cdots & 0 & 0 \\
-\beta_{n-1} \omega_{o}^{n-1}+r_{n-1} & \vdots & \vdots & \ddots & \vdots & \vdots \\
-\beta_{n} \omega_{o}^{n}+r_{n} & -k_{1} & -k_{2} & \cdots & -k_{n-1} & 1 \\
-\beta_{n+1} \omega_{o}^{n+1} & 0 & 0 & \cdots & 0 & 0
\end{array}\right] .
$$


Now, for the system given in (38), a following change of coordinates is introduced: $q_{1}=\omega_{o}^{-n} \xi_{1}, q_{2}=\omega_{o}^{-(n-1)} \xi_{2}, \ldots, q_{n+1}=\xi_{n+1}$, with its general form $q_{i}=\omega_{o}^{i-(n+1)} \xi_{i}$, for $i=1,2, \ldots, n+1$, or expressed alternatively in matrix representation as $\boldsymbol{q}=\boldsymbol{\Lambda} \xi$, with $\boldsymbol{\Lambda}=\operatorname{diag}\left[\omega_{o}^{-n} \omega_{o}^{-(n-1)} \cdots \omega_{o}^{-1} 1\right]$ and $\xi=\left[\begin{array}{lllll}\xi_{1} & \xi_{2} & \cdots & \xi_{n} & \xi_{n+1}\end{array}\right]$. This allows to rewrite (38) as:

$\boldsymbol{\Lambda} \dot{\xi}=\left(A-l c^{\top}\right) \Lambda \xi+h \eta=H \Lambda \xi+h \eta$,

which yields:

$\frac{1}{\omega_{o}} \dot{\xi}=\boldsymbol{H}_{q} \boldsymbol{\xi}+\frac{1}{\omega_{o}} \boldsymbol{h} \eta \Longrightarrow \varepsilon \dot{\xi}=\boldsymbol{H}_{q} \boldsymbol{\xi}+\varepsilon \boldsymbol{h} \eta$,

with $\varepsilon=\frac{1}{\omega_{o}}$, and $\boldsymbol{H}_{q}=\boldsymbol{A}_{q}+\varepsilon \boldsymbol{H}_{\varepsilon}$, where:

$\boldsymbol{A}_{q}=\left[\begin{array}{cccccc}-\beta_{1} & 1 & 0 & \cdots & 0 & 0 \\ -\beta_{2} & 0 & 1 & \cdots & 0 & 0 \\ \vdots & 0 & 0 & \cdots & 0 & 0 \\ -\beta_{n-1} & \vdots & \vdots & \ddots & \vdots & \vdots \\ -\beta_{n} & 0 & 0 & \cdots & 0 & 1 \\ -\beta_{n+1} & 0 & 0 & \cdots & 0 & 0\end{array}\right]$,

$\boldsymbol{H}_{\varepsilon}=\left[\begin{array}{cccccc}r_{1} & 0 & 0 & \cdots & 0 & 0 \\ \varepsilon r_{2} & 0 & 0 & \cdots & 0 & 0 \\ \vdots & 0 & 0 & \cdots & 0 & 0 \\ \varepsilon^{n-2} r_{n-1} & \vdots & \vdots & \ddots & \vdots & \vdots \\ \varepsilon^{n-1} r_{n} & -\varepsilon^{n-2} k_{1} & -\varepsilon^{n-3} k_{2} & \cdots & -k_{n-1} & 0 \\ 0 & 0 & 0 & \cdots & 0 & 0\end{array}\right]$,

with $\beta_{i}$ 's and $k_{i}$ 's being defined in (42). Also, $r_{i}$ 's are functions of $\omega_{o}, \beta_{i}$ 's and $k_{i}$ 's, and they are completely determined in (43).

With above alternative representation, a proper stability analysis of the general error-based compact ADRC can now be performed. First, the observer error dynamics (46) with its initial condition writes:

$\varepsilon \dot{\xi}=\boldsymbol{H}_{q} \xi+\varepsilon h \cdot \eta, \quad \xi(0)=\xi_{0}$.

It is an initial value problem of a standard singularly perturbed linear system of differential equations with a small positive parameter $\varepsilon=\frac{1}{\omega}$, that depends on the observer bandwidth $\omega_{o}$. Note, that matrix $\boldsymbol{H}_{q}$ and the estimation-error state matrix $\boldsymbol{H}$ are similar $\left(\boldsymbol{H}=\boldsymbol{\Lambda} \boldsymbol{H}_{q} \boldsymbol{\Lambda}^{-1}\right)$ and have the same eigenvalues. Thus, $\lambda_{i}\left(\boldsymbol{H}_{q}\right)=\lambda_{i}(\boldsymbol{H})=-\omega_{o}$ and $\lambda_{i}\left(\boldsymbol{A}_{q}\right)=-1$, $\forall i=1,2, \ldots, n+1$, which implies $\boldsymbol{H}_{q}$ is Hurwitz. As in Shao and Gao (2017), it can be concluded that for a quite large class of total disturbance $f^{*}$, if $\left\|\xi_{0}\right\|=O(\varepsilon)$, i.e. $\xi_{0}$ lies in the stable initial manifold, there will be no danger of large magnitude transients in system (49) as $\varepsilon \rightarrow 0$ for $t>0$, and same system is exponentially stable and uniformly asymptotically stable (for stability within the stable initial manifold for $\xi_{0}$ - it is not the stability in the Lyapunov sense). In the spirit of Theorems 3.1 and 4.1 of Shao and Gao (2017), main stability theorem is obtained as below:

Theorem 1. If $\left\|\xi_{0}\right\|=O(\varepsilon)$ and $\eta=\dot{f}^{*}$ exists, then:

(i) the asymptotic solution of (49),

$\varepsilon \dot{\xi}=\boldsymbol{H}_{q} \xi+\varepsilon h \cdot \eta=\left(\boldsymbol{A}_{q}+\varepsilon \boldsymbol{H}_{\varepsilon}\right) \xi+\varepsilon h \cdot \eta, \xi(0)=\xi_{0}$,

is uniformly valid for all finite time $L$ with $0 \leq t \leq L<\infty$, and it has the following form:

$$
\begin{aligned}
\xi(\varepsilon, t) & =\exp \left(\boldsymbol{A}_{q} \frac{t}{\varepsilon}\right) \xi_{0}+\varepsilon\left[\boldsymbol{A}_{q}^{-1} h \cdot \eta+\exp \left(\boldsymbol{A}_{q} \frac{t}{\varepsilon}\right)\left\{\boldsymbol{A}_{q}^{-1} h \cdot \eta(0)\right\}\right. \\
& \left.-\boldsymbol{H}_{0} \int_{0}^{t} \exp \left[\boldsymbol{A}_{q}\left(\frac{t-s}{\varepsilon}\right)\right] \boldsymbol{A}_{q}^{-1} h \cdot \eta(s) d s\right]+O\left(\varepsilon^{2}\right) ;
\end{aligned}
$$

(ii) there exists $\varepsilon^{*}>0$ such that for all $\varepsilon \in\left[0, \varepsilon^{*}\right]$ the system (49) is exponentially stable as $\varepsilon \rightarrow 0$;

(iii) for all $\xi \in \Omega=\left[-\rho_{1}, \rho_{2}\right]^{n+1} \subset \mathbb{R}^{n+1}$ with $\rho_{2}=\gamma \max \left\{\omega_{c}, \frac{1}{\omega_{o}},\left\|\boldsymbol{H}_{q}\right\|\right\}$ for some constant $\gamma>0$, then there exists positive constant $C_{2}$, independent of $\varepsilon$, and the solution of (49) satisfies:

$\|\xi(\varepsilon, t)\| \leq \varepsilon C_{1} \exp \left[-\left(\frac{1}{2}-\varepsilon C_{1} C_{2}\right) \frac{t}{\varepsilon}\right],\|\eta\| \cdot\|\xi(\varepsilon, t)\|^{-1} \leq C_{2}$, where $C_{1}=\sqrt{n+1}+\frac{1}{\omega_{c}} \sum_{j=1}^{n} \frac{L^{j}}{j !}\left\|\left(\boldsymbol{H}_{q}+\boldsymbol{I}_{n+1}\right)^{j^{2}}\right\|$ with $\boldsymbol{I}_{n+1}$ is the $n+1$ order identity matrix and:

$\boldsymbol{H}_{0}=\left[\begin{array}{cccccc}n \omega_{c}^{n-1} & 0 & 0 & \cdots & 0 & 0 \\ \vdots & \vdots & \vdots & \ddots & \vdots & \vdots \\ 0 & 0 & 0 & \cdots & -n \omega_{c}^{n-1} & 0 \\ 0 & 0 & 0 & \cdots & 0 & 0\end{array}\right]$.

Comments: ad.(i) Theorem 1 states that the system (49) is exponentially stable and uniformly asymptotically stable if $\left\|\xi_{0}\right\|=O(\varepsilon)=$ $O\left(\frac{1}{\omega_{o}}\right)$ and $\eta=\dot{f}^{*}$ exists (the result holds, for stability within the stable initial manifold for $\xi_{0}$, it is not the stability in the Lyapunov sense). This means that the stability results in Shao and Gao (2017) can be extended to the proposed error-based compact ADRC, which the error dynamic system (49) has a more general coefficient matrix $\boldsymbol{H}_{q}=\boldsymbol{A}_{q}+\varepsilon \boldsymbol{H}_{\varepsilon}$; ad.(ii) If the total disturbance $f^{*}$ is a function of Sobolev class $W_{\text {loc }}^{1,1}$ and differentiable a.e. (an a.e. differentiable function is a function that is differentiable except on a set of measure zero), then its weak derivative is represented by a pointwise derivative (for details see Evans \& Gariepy, 1991), thus all the results in Theorem 1 hold.

\section{References}

Chu, Z., Sun, Y., Wu, C., \& Sepehri, N. (2018). Active disturbance rejection control applied to automated steering for lane keeping in autonomous vehicles. Control Engineering Practice, 74, 13-21. http://dx.doi.org/10.1016/j.conengprac.2018.02.002.

Cortes-Romero, J., Jimenez-Triana, A., Coral-Enriquez, H., \& Sira-Ramirez, H. (2017). Algebraic estimation and active disturbance rejection in the control of flat systems. Control Engineering Practice, 61, 173-182. http://dx.doi.org/10.1016/j.conengprac. 2017.02.009.

Dai, C., Yang, J., Wang, Z., \& Li, S. (2017). Universal active disturbance rejection control for non-linear systems with multiple disturbances via a high-order sliding mode observer. IET Control Theory Applications, 11(8), 1194-1204. http://dx.doi.org/10. 1049/iet-cta.2016.0709.

Evans, L. C., \& Gariepy, R. F. (1991). Measure theory and fine properties of functions. CRC Press.

Fliess, M., \& Join, C. (2013). Model-free control. International Journal of Control, 86(12), 2228-2252. http://dx.doi.org/10.1080/00207179.2013.810345.

Gao, Z. (2003). Scaling and bandwidth-parameterization based controller tuning. In American control conference, vol. 6 (pp. 4989-4996). http://dx.doi.org/10.1109/ACC. 2003.1242516.

Gao, Z. (2006). Active disturbance rejection control: a paradigm shift in feedback control system design. In American control conference (pp. 2399-2405). http://dx.doi.org/10. 1109/ACC.2006.1656579.

Gao, Z. (2014). On the centrality of disturbance rejection in automatic control. ISA Transactions, 53(4), 850-857. http://dx.doi.org/10.1016/j.isatra.2013.09.012.

Godbole, A. A., Kolhe, J. P., \& Talole, S. E. (2013). Performance analysis of generalized extended state observer in tackling sinusoidal disturbances. IEEE Transactions on Control Systems Technology, 21(6), 2212-2223. http://dx.doi.org/10.1109/TCST. 2012.2231512.

Guerra, A. D. L., Arteaga-Perez, M. A., Gutierrez-Giles, A., \& Maya-Ortiz, P. (2016). Speedsensorless control of SR motors based on GPI observers. Control Engineering Practice, 46, 115-128. http://dx.doi.org/10.1016/j.conengprac.2015.10.010.

Han, J. (2009). From PID to active disturbance rejection control. IEEE Transactions on Industrial Electronics, 56(3), 900-906. http://dx.doi.org/10.1109/TIE.2008.2011621.

Herbst, G. (2016). Practical active disturbance rejection control: bumpless transfer, rate limitation, and incremental algorithm. IEEE Transactions on Industrial Electronics, 63(3), 1754-1762. http://dx.doi.org/10.1109/TIE.2015.2499168.

Kokotovic, P., Khalil, H. K., \& O’Reilly, J. (1999). Singular perturbation methods in control: analysis and design. SIAM, http://dx.doi.org/10.1137/1.9781611971118.

Lotfi, N., Zomorodi, H., \& Landers, R. G. (2016). Active disturbance rejection control for voltage stabilization in open-cathode fuel cells through temperature regulation. Control Engineering Practice, 56, 92-100. http://dx.doi.org/10.1016/j.conengprac. 2016.08.006.

Madonski, R., Gao, Z., \& Lakomy, K. (2015). Towards a turnkey solution of industrial control under the active disturbance rejection paradigm. In Conference of the society of instrument and control engineers of Japan (pp. 616-621). http://dx.doi.org/10.1109/ SICE.2015.7285478.

Madonski, R., Kordasz, M., \& Sauer, P. (2014). Application of a disturbance-rejection controller for robotic-enhanced limb rehabilitation trainings. ISA Transactions, 53(4), 899-908. http://dx.doi.org/10.1016/j.isatra.2013.09.022.

Madonski, R., Nowicki, M., \& Herman, P. (2014). Application of active disturbance rejection controller to water supply system. In Chinese control conference (pp. 40014005). http://dx.doi.org/10.1109/ChiCC.2014.6895677.

Madonski, R., Nowicki, M., \& Herman, P. (2016). Practical solution to positivity problem in water management systems - an ADRC approach. In American control conference, (pp. 1542-1547). http://dx.doi.org/10.1109/ACC.2016.7525135. 
Michalek, M. M. (2016). Robust trajectory following without availability of the reference time-derivatives in the control scheme with active disturbance rejection. In American control conference (pp. 1536-1541). http://dx.doi.org/10.1109/ACC.2016.7525134.

Michalek, M. M., \& Kielczewski, M. (2017). Robustification of the modular tracking control system for non-standard n-trailers of uncertain kinematics. Control Engineering Practice, 64, 160-172. http://dx.doi.org/10.1016/j.conengprac.2017.04.005.

Nowak, P., Stebel, K., Klopot, T., Czeczot, J., Fratczak, M., \& Laszczyk, P. (2018). Flexible function block for industrial applications of active disturbance rejection controller. Archives of Control Sciences, 28(3), 379-400. http://dx.doi.org/10.24425/acs.2018. 124708.

Ramirez-Neria, M., Sira-Ramirez, H., Garrido-Moctezuma, R., \& Luviano-Juarez, A. (2014). Linear active disturbance rejection control of underactuated systems: the case of the Furuta pendulum. ISA Transactions, 53(4), 920-928. http://dx.doi.org/ 10.1016/j.isatra.2013.09.023.

Shao, S., \& Gao, Z. (2017). On the conditions of exponential stability in active disturbance rejection control based on singular perturbation analysis. International Journal of Control, 90(10), 2085-2097. http://dx.doi.org/10.1080/00207179.2016.1236217.

Sira-Ramirez, H., \& Silva-Ortigoza, R. (2006). Control design techniques in power electronics devices. London: Springer-Verlag, http://dx.doi.org/10.1007/1-84628-459-7.
Using the UCD9224EVM-464 - user's guide. (2017). Texas Instruments, website: ti.com.

Wang, Z., Li, S., Wang, J., \& Li, Q. (2017). Robust control for disturbed buck converters based on two GPI observers. Control Engineering Practice, 66, 13-22. http://dx.doi. org/10.1016/j.conengprac.2017.06.001.

Wu, G., Sun, L., \& Lee, K. Y. (2017). Disturbance rejection control of a fuel cell power plant in a grid-connected system. Control Engineering Practice, 60, 183-192. http: //dx.doi.org/10.1016/j.conengprac.2016.12.010.

Xue, W., \& Huang, Y. (2015). Performance analysis of active disturbance rejection tracking control for a class of uncertain LTI systems. ISA Transactions, 58, 133-154. http: //dx.doi.org/10.1016/j.isatra.2015.05.001.

Xue, W., \& Huang, Y. (2018). Performance analysis of 2-DOF tracking control for a class of nonlinear uncertain systems with discontinuous disturbances. International Journal of Robust Nonlinear Control, 28, 1456-1473. http://dx.doi.org/10.1002/rnc.3972.

Xue, W., Madonski, R., Lakomy, K., Gao, Z., \& Huang, Y. (2017). Add-on module of active disturbance rejection for set-point tracking of motion control systems. IEEE Transactions on Industry Applications, 53(4), 4028-4040. http://dx.doi.org/10.1109/ TIA.2017.2677360.

Zhang, H. (2017). Information driven control design: a case for PMSM control (Doctoral dissertation), Cleveland State University.

Zheng, Q., Chen, Z., \& Gao, Z. (2009). A practical approach to disturbance decoupling control. Control Engineering Practice, 17(9), 1016-1025. http://dx.doi.org/10.1016/j. conengprac.2009.03.005. 